\title{
¿INDÍGENAS TRANSNACIONALES O NACIONALES? \\ TENSIONES ENTRE EL CARNAVAL ANDINO DE ARICA Y EL DESARROLLO TURÍSTICO EN LA FRONTERA
}

\author{
National or Transnational Indigenous People? Tensions Between the Andean \\ Carnival of Arica and Turism Development in the Border
}

\section{DANTE CHOQUE-CASERES*}

Fecha de recepción: 25 de mayo de 2019 - Fecha de aprobación: 20 de agosto de 2019

\section{Resumen}

El presente artículo tiene como objetivo describir los conflictos que han emergido en el desarrollo turístico y las expresiones culturales transfronterizas de base indígena que se manifiestan en la urbanidad de la zona fronteriza. Tomando como caso el Carnaval Andino de Arica, se observa que las prácticas indígenas reinventadas y reterritorializadas tensionan la identidad y la cultura dominante nacional. Para los organizadores, el desarrollo del carnaval debe alinearse con los intereses turísticos establecidos por las autoridades centrales a través de la estructura institucional y de planificación. Basado en un proceso de observación de las diferentes versiones del carnaval entre 2013 y 2019, junto con entrevistas a diversos actores, los resultados demuestran que el desarrollo turístico del evento ha contribuido a la negación implícita de los vínculos de los indígenas de Arica con los países vecinos.

Palabras clave: turismo indígena; pueblos indígenas; Carnaval Andino de Arica; soberanía cultural.

\section{Abstract}

The present paper aims to describe the conflicts that have arisen between tourism development and Indigenous People' culture-based expressions in a borderland city. Taking the Andean Carnival of Arica as a case, the reinvented and reterritorialized Indigenous practices conflict with the national identity and culture. For organizers, the development of Carnival should be aligned with the tourist interests defined by central authorities through the institutional and planning structure. Based on the observation of the Carnival between 2013 and 2019 along with interviews of key informants, the results show that the tourism development has contributed to the rejection of the ties between local Indigenous people and neighbouring countries.

Keywords: indigenous tourism; indigenous peoples; Andean Carnival of Arica; cultural sovereignty.

* Dr. en Estudios Latinoamericanos. Investigador asociado al Centro de Estudios Interdisciplinarios e Indígenas (CIIR), Arica, Chile. Correo-e: dchoquec@gmail.com 


\section{Introducción}

El Carnaval Andino de Arica "Con la Fuerza del Sol" es un evento que pronto cumplirá veinte años desde su primera versión. Dada la cantidad de bailarines, músicos, turistas y público que congrega, es promocionado a nivel nacional como uno de los carnavales más grandes de Latinoamérica luego del Carnaval de Río de Janeiro en Brasil y el Carnaval de Oruro en Bolivia. A diferencia de estos carnavales, los bailarines y los músicos organizados en comparsas o fraternidades compiten durante tres días en uno de dos grupos de categorías: danzas internacionales y danzas de rescate local. En la primera están consideradas el caporal, la morenada, el tinku, el waka-waka, la diablada, entre otras, mientras que la segunda corresponde a los bailes de los pueblos ancestrales, afrodescendientes y también las tarqueadas. Los participantes son coordinados por dos organizaciones: la Federación de la Cultura y las Artes Indígenas Kimsa Suyu y la Asociación Indígena Confraternidad de Agrupaciones Folclóricas Andinas de Arica Inti Ch'amampi. Ellas, junto con la Municipalidad de Arica, son quienes realizan la producción general del evento a través de una comisión organizadora, la cual planifica, elabora y coordina todas las actividades.

Este evento conecta a Bolivia, Chile y Perú, los cuales participaron en la Guerra del Pacífico (1879-1883), y tensiona un territorio con fronteras regularmente nacionalizadas dado su valor geopolítico. En el caso de Chile, las autoridades cívicas, religiosas y militares conmemoran anualmente la Batalla de Arica o Asalto y Toma del Morro de Arica ocurrida el 7 de junio de 1880. Ese día Chile derrotó a Perú, con lo cual ocupó militarmente este territorio, que luego anexó a su soberanía en un proceso que terminó en 1929 con el Tratado de Lima. Es tan importante esta fecha que fue declarada en 2013 feriado regional en respuesta a una demanda local ${ }^{1}$.

A pesar de los procesos de chilenización, el reforzamiento de la soberanía en la región es presentado como una necesidad en los discursos oficiales dado los conflictos legales que Chile ha enfrentado en la Corte Internacional de Justicia de La Haya. A la fecha, el gobierno ha sido demandado tres veces: uno por Perú (2008-2014) y dos por Bolivia (20132018 y 2016-presente). En este sentido, el actual gobierno le encargó al Ministerio de Relaciones Exteriores la elaboración de un plan maestro de desarrollo para las comunas rurales, las cuales han sido afectadas por el fenómeno del despoblamiento (Ruiz, 2018). En este contexto, una atmósfera negativa debería aparecer en contra del desarrollo de cualquier manifestación sociocultural que involucre las nacionalidades boliviana y peruana.

Dado el componente extranjero, este evento es constantemente criticado a nivel local. En redes sociales, el carnaval es renombrado como "Carnaval Boliviano" y, junto con ello, son resaltados los efectos negativos del evento, como las peleas, la suciedad y el consumo de alcohol, haciendo una diferencia respecto de la llamada cultura chilena. Por otra parte, grupos de personas han solicitado su suspensión en diferentes ocasiones. En 2017, el argumento fue los incendios forestales que se generaron en múltiples regiones de la zona centro y sur de Chile. En 2019, el motivo era los efectos de las lluvias estivales tanto en la ciudad como en los pueblos del interior ${ }^{2}$. Estos hechos sugieren que es necesario considerar 
que la percepción de los residentes locales ha cambiado de acuerdo con el ciclo de desarrollo y el involucramiento en la actividad turística en torno al evento (Woo, Uysal \& Sirgy, 2019). Para justificar la realización del carnaval, un razonamiento utilizado por la organización ha sido el impacto económico del evento en la región, puesto que permite incrementar tanto la llegada como el gasto de los turistas en el comercio local. Al finalizar la última versión, la Municipalidad de Arica estimó en 1.500 millones de pesos los gastos de turistas por alojamiento, alimentación y otros servicios durante los días de carnaval (Mamani, 2019).

En este contexto, dos discursos emergen y colisionan en torno al desarrollo del Carnaval Andino de Arica. El primero revela la relación de Arica con los territorios circundantes más allá de las fronteras. Dicho discurso es coherente con el hecho de que la región de Arica y Parinacota ha sido históricamente parte de un comercio de intercambios, una migración circular y un movimiento político indígena que se ha dado entre la cordillera y la costa. Un segundo discurso incrusta el evento dentro de un marco atemporal, homogenizado y despolitizado, que facilita su incorporación en una agenda de desarrollo turístico. Tanto para los dirigentes de las agrupaciones de baile como para las autoridades regionales y nacionales, el Carnaval Andino de Arica es un recurso que debe ser posicionado para favorecer el desarrollo de un destino turístico. El Carnaval Andino de Arica, al constituirse como un evento temporal y, en especial, peculiar, tendría un valor para esta actividad económica.

El hecho que el carnaval es una competencia donde hay ganadores y perdedores parece relegado al segundo plano. Emerge, al centrarse en este aspecto, el control de la autenticidad, la integridad y la aceptabilidad como una instancia en la cual se apoyan, niegan u obvian ciertas características del carnaval. Aunque estos tres valores otorgan a los pueblos indígenas una posibilidad de autodeterminación de sus tradiciones (Theodossopoulos, 2010), la organización controla el evento. Al respecto, aunque De la Cadena (2009) afirma que los pueblos indígenas han diseñado formas no convencionales para alcanzar sus demandas de autodeterminación, en este caso, la institucionalidad creada en torno al carnaval ubica los discursos sobre este aspecto únicamente en la perpetuación de las tradiciones culturales. De esta forma, hacia fuera de la región, el carnaval es presentado en coherencia con las aspiraciones expresadas en la planificación estratégica del turismo y se destaca de sobremanera el rescate de las prácticas culturales ancestrales que pueden ser asociadas con Chile.

Aunque la mayoría de las comparsas presentan bailes que no tienen un origen en Chile, la propaganda turística destaca lo indígena y/o afrodescendiente circunscrito no a las fronteras, sino al territorio chileno, con lo cual se omiten las relaciones transnacionales. De esta forma, este ápice de la cultura aymara-quechua o, más bien, andina, que ha sobrevivido a los diferentes procesos de chilenización se muestra minúsculo, independiente y único, lo que conlleva a resignificarlo como una expresión cultural del "Chile profundo" o de la "América profunda"3. Por lo tanto, la hipótesis aquí planteada es que el desarrollo turístico del carnaval ha contribuido a la invisibilización de las demandas políticas, económicas y sociales de los pueblos indígenas. 
El presente artículo analiza el desarrollo del Carnaval Andino de Arica desde la intersección de dos perspectivas: la transnacional y la de desarrollo turístico. El estudio considera la observación del carnaval entre 2013 y 2018. La fecha exacta de realización varía año a año: los organizadores han tomado como regla que el carnaval se efectúe 40 días antes de la semana Santa, lo cual permite la participación de los bailarines, si así lo quisieran, en el Carnaval de Oruro. En cada ocasión anual se hizo una observación de los tres días que dura el carnaval y de las actividades complementarias, por ejemplo, el lanzamiento del carnaval. La observación de las diferentes actividades fue registrada mediante fotografías, las cuales permiten percibir la forma en que son construidas las identidades y los espacios, lo que favorece la reflexión pausada de eventos que por su ejecución ocurren rápidamente.

Además, puesto que las comparsas participan todos los años, fue posible identificar la manera en que ha evolucionado la simbología a través de las diferentes versiones. Además de estos aspectos, también fueron incorporadas otras fuentes de información: la documentación oficial de la organización del Carnaval; notas de prensa de diarios locales como La Estrella de Arica, ex ante, ex dure y ex post evento, y entrevistas a los presidentes de la Federación de la Cultura y las Artes Indígenas Kimsa Suyu y de la Asociación Indígena Confraternidad de Agrupaciones Folclóricas Andinas de Arica Inti Ch'amampi y a dirigentes de comparsas en la versión 2015 del carnaval. Las preguntas en las entrevistas fueron abiertas y su principal tema fueron los cambios que han ocurrido en este evento en los últimos años.

\section{Transnacionalidad e intersección con el turismo}

Parte de las danzas del carnaval tiene un origen en Bolivia. Se puede suponer que fueron introducidas por comunidades de migrantes para luego ser adoptadas por la población nacional. No obstante, para algunos es importante que las autoridades mantengan el control de la imagen del carnaval fuera del espacio regional. En este contexto, un enfoque transnacional sería adecuado para describir las tensiones identitarias y culturales que emergen en el evento.

Desde comienzos de la década de 1990, la transnacionalidad surge como un enfoque en los estudios migratorios (Dahinden, 2012; Derichs, 2017). Esta propuesta sugiere colapsar ciertas concepciones binarias y jerárquicas, tales como país de origen vs destino, emigración vs inmigración, entre otras (Faist, 2008). De esta forma, esta teoría instala a los migrantes en un tercer espacio (Bhabha, 2014), que implica el vivir en forma simultánea en el lugar de origen y en el de destino. Este espacio de contradicción y ambivalencia subordina la construcción de una nueva identidad, como tema central, al proceso de ponderación creativa de voces, narrativas e historias a partir de la coexistencia de una cultura dominante y múltiples vulnerables. En este caso, la transnacionalidad será entendida como "la emergencia de nuevas formas de vida y acción cuya lógica interna es explicada por la capacidad inventiva de los humanos para crear y mantener contextos y relaciones sociales de intercambio sin medir distancias" (Cuninghame, 2008, p. 25). El análisis desde esta perspectiva se concentra en la complejidad de la movilidad sociocultural a través de las fronteras nacio- 
nales que produce sujetos que son capaces de reproducir la atmósfera y la cultura de sus lugares de origen.

Desde esta perspectiva, la transnacionalidad explora el desarrollo de un sentido de experiencia en común, que no siempre está conectada a significantes culturales, lugares de origen 0 identidades étnicas (Brown, 2013). En este último punto, a diferencia de los estudios de la diáspora, el enfoque transnacional enfatiza el análisis de la movilidad a través de las fronteras sobre la construcción de identidades colectivas (Faist, 2010, p. 21). Respecto a esto, se considera que la población migrante puede acceder a múltiples identidades, las cuales son instrumentalizadas como parte de una estrategia de sobrevivencia para alcanzar un mejor bienestar. En otras palabras, el interés se encuentra en "las formas de ser" más que en "las formas de pertenecer".

En este marco, existen dos aspectos que son escasamente abordados en los estudios transnacionales. El primero se refiere a la preferencia por la problematización desde experiencias en ciudades globales (ver p. ej. Plüss, 2018), relegando los análisis en zonas fronterizas. Al respecto, aunque se pudiera pensar que las decisiones sobre lo que ocurre en la zona fronteriza son determinadas solamente por las autoridades centrales, la gobernanza es más compleja puesto que tanto autoridades como residentes locales tienes diferentes intereses en el territorio.

Un segundo aspecto consiste en la incorporación de la variable indígena en los análisis sobre comunidades y espacios transfronterizos. Jean (2007) sugiere reconocer cómo el hecho de pertenecer a una comunidad indígena mayor (transnacional o global) permitiría superar los límites establecidos por la estratificación legal del país de destino. Generalmente, el desarrollo de proyectos transfronterizos con base en la identidad o cultura indígena es limitado por el control de los agentes estatales. Considerando estos aspectos, la teoría transnacional debería explicar, en este caso, cómo las comunidades y espacios transnacionales reconfiguran los Estados-nación y no solamente la emergencia de nuevas formas de ciudadanía.

La comunidad transnacional trasciende esencialmente a lo local o nacional en términos organizativos y es capaz de mantener un intercambio continuo de bienes, información, ideas, memorias y también personas. Faist (2013) sugiere evitar etiquetar a los migrantes solamente utilizando categorías étnicas o nacionales y enfocarse en los procesos de formación social, por ejemplo, las comunidades de migrantes que constituyen redes de reciprocidad en los mercados fronterizos. Así, también, la comunidad no es exclusiva de migrantes, sino que considera además a las poblaciones locales que son contiguas, quienes en conjunto tienen la capacidad de reubicarse en una sola frontera y promover la participación de migrantes. Sus miembros dejan de considerar ciertas distinciones identitarias aprovechando el acceso a diversas redes para desarrollar procesos a través de las fronteras. Cabe mencionar que la comunidad transnacional es una red transnacional, pero lo contrario no siempre es válido (Djelic \& Quack, 2010). La comunidad actuaría a partir del reconocimiento de un conjunto de intereses en común que permite articular un espacio social transnacional. 
La formación de un espacio transnacional requiere de la circulación y la interacción recurrente de discursos y prácticas a través de las fronteras, las cuales activan o desactivan ciertas ideas, símbolos, actividades y cultura material. De acuerdo con Crang, Dwyer y Jackson (2003), este espacio se caracteriza por ser complejo, multidimensional y habitado por un rango amplio de actores. En particular, la noción de multidimensional deriva de la superposición de lo local, global y transnacional. La comunidad transnacional sería, entonces, capaz de crear flujos y relaciones que llevan a la emergencia de estos espacios que transforman lugares.

Respecto del vínculo espacio y lugar es necesario considerar que Lefebvre (1991 [1974]) propone analizar la producción del espacio incluyendo las prácticas espaciales, la representación del espacio y los espacios de representación. De acuerdo con esta propuesta, el espacio es simultáneamente un campo de acción y una base para la acción. Mirado así, la ciudad como lugar cobra relevancia para la actividad transfronteriza. En este marco, los migrantes son sujetos activos y están conectados materialmente con oportunidades económicas, estructuras políticas y prácticas culturales. Este fenómeno se puede explorar con mayor profundidad en la conceptualización del urbanismo transnacional, tema que va más allá del objetivo de este artículo (ver Smith, 2001).

En la ciudad, los discursos sobre el diseño y la construcción de lugares que emergen desde las comunidades transnacionales (Smith, 2017) pueden ser entendidos como un place-making. Este proceso permite representar un espacio transnacional como una expresión geográfica de la comunidad. Para Richards (2017), el proceso de place-making es holístico puesto que emplea no solo recursos materiales, sino también símbolos y conceptos que le dan significado a un lugar en función de las expectativas de diversos grupos de interés. En este aspecto, el intercambio simbólico y material es un recurso clave para promover proyectos de desarrollo comunitario a través de las fronteras en diferentes escalas. Ejemplos, en este aspecto, son los barrios chinos o chinatowns que se localizan dentro de las ciudades, que transforman los lugares y que permiten a los migrantes participar simultáneamente en diferentes redes de influencia. En este aspecto también es necesario considerar los pequeños eventos culturales organizados por migrantes, que pueden jugar un rol importante en los procesos de place-making (Fisker, Kwiatkowski \& Hjalager, 2019).

El punto de intersección entre el transnacionalismo y la actividad turística es el lugar, el cual tiene mucho valor para el turismo puesto que es preponderante para el desarrollo de tal experiencia. La actividad turística puede reforzar ciertos significados y representaciones en la construcción social del lugar, que derivan en un proceso de place-making. Desde el turismo, la intervención de un lugar está relacionada con el fortalecimiento de las ventajas competitivas de un barrio, ciudad o país. En este marco, quienes administran el turismo tienen la capacidad de coaptar comunidades y sus lugares con el fin de desarrollar productos turísticos para ser incorporados a las actividades de marketing. El objetivo, en este caso, es desarrollar algún grado de autenticidad para que un lugar pueda ser consumido por el turista, es decir, las personas internalizan emocionalmente memorias colectivas para afianzar una experiencia individual (Lovell \& Bull, 2018). 
Por otra parte, la convergencia entre el transnacionalismo y la actividad turística no es directa, sino más bien pasa por la caracterización del residente y el turista. Aunque en el pasado se consideraban estos sujetos como opuestos, en particular al tomar en cuenta su interacción con el lugar, es decir, entre hacer y deshacer un lugar, hoy tal distinción parece ser menos válida (Chang, 2012). En especial, el turista posee, en algunos casos, más información sobre un lugar que los mismos residentes, y estos últimos influyen en la forma en que se desarrollan las prácticas del turismo. Los roles de residentes y turistas pueden ser reexaminados agregando una capa más en la complejidad, partiendo por establecer que el conocimiento del turista está influenciado por la autoridad turística local y que el residente podría ser un sujeto transnacional que influye de cierta forma en el desarrollo del turismo. Estos razonamientos serán desarrollados en los siguientes apartados.

\section{El turismo como estrategia de desarrollo}

El turismo impulsó el crecimiento de Arica con mucha anterioridad a su creación como región. Entre 1930 y 1975, el territorio circunscrito en la actual región de Arica y Parinacota conformaba el departamento de Arica, que era parte de la provincia de Tarapacá. En este período el gobierno crea la Junta de Adelanto de Arica (1958-1976), una institución público-privada, que fue el principal promotor del turismo, porque la ley definió que sus ingresos provenían de los impuestos recaudados en el departamento. Basado en la necesidad de fomentar el comercio y turismo local, entre otras medidas, se estableció como objetivo construir, ampliar y mejorar un conjunto de infraestructura pública vinculada al turismo, entre ellas, el casino de Arica, la playa la Lisera, el aeropuerto Chacalluta, el terminal rodoviario, el estadio Carlos Dittborn, entre otros. Junto con ello, se patrocinaron diversos eventos turísticos como, por ejemplo, el Concurso Reina de las Playas de Chile. De tal forma, el turismo se concentró exclusivamente en la ciudad marginando el resto del departamento.

En 1963, el interés de la autoridad se volcó sobre los pueblos del interior con la incorporación del departamento de Arica en el Andean Indian Program de la Organización de las Naciones Unidas (ONU), el cual estaba siendo ejecutado desde 1952 en Bolivia, Ecuador y Perú. Posteriormente, en 1974, el Comité de Desarrollo del Interior, el cual incluyó a todas las organizaciones públicas en el departamento, definió un conjunto de iniciativas para continuar con el desarrollo de los pueblos del interior y, sobre todo, para afianzar la soberanía chilena. El Comité solicitó un diagnóstico a la Junta de Adelanto de Arica, el cual mencionaba que

[...] respecto del recurso humano, que en general, es de bajo standard tecnológico y por lo tanto su rendimiento económico también es bajo. Ha permanecido por varios años estancado en un sistema de producción limitado que no resultan en ningún modo favorables a las exigencias de la época (Junta de Adelanto de Arica, 1974, p. 13).

Este diagnóstico establece una brecha basada en una perspectiva económica y productiva en evidente comparación con el desarrollo experimentando en la ciudad de Arica. De esta forma, el espacio para discursos alternativos que prioricen las comunidades, el medioambiente o los medios de vida fueron relegados. La zona rural de la región fue asociada al turismo de manera muy puntual, por ejemplo, se consideraron como atractivos turísticos la artesanía, 
las iglesias y las festividades autóctonas. Sin embargo, este proyecto no logró concluirse debido al proceso de regionalización (19741976), el cual terminó con la Junta de Adelanto y transformó el departamento en las provincias de Arica y Parinacota, dependientes de la región de Tarapacá. El impulso público del turismo se diluyó severamente en este nuevo esquema político-administrativo.

En este período se acentuó el despoblamiento del altiplano, con lo cual las festividades asociadas a la religiosidad aymara, entre ellas los carnavales de los pueblos del interior, comenzaron a disminuir en su envergadura puesto que eran practicadas por pocas familias (Gavilán \& Carrasco, 2009). En este nuevo contexto, la relación que destaca Leal (2017) entre prácticas rituales y espacios sagrados comienza a debilitarse. Como parte de la memoria colectiva, dichas prácticas empiezan a emerger en el plano urbano de la ciudad de Arica.

Existe una larga pausa para el desarrollo del turismo hasta la creación de la región de Arica y Parinacota en 2008, con lo cual se va estableciendo progresivamente una estructura de planificación para que la región sea un destino turístico. Por una parte, el gobierno central impulsó el Plan de Desarrollo Turístico de Arica y Parinacota 2011-2014, y, por la otra, el gobierno regional de Arica y Parinacota formuló y aprobó la Política Regional de Turismo 2012-2016. Luego, el Servicio Nacional de Turismo elaboró un Plan de Acción de Turismo de Arica y Parinacota 2014-2018. Cada uno de estos planes identificó iniciativas que se pueden enmarcar en diferentes ámbitos del turismo, como: desarrollo de la oferta, promoción y difusión, infraestructura, desarrollo de alianzas, entre otros. Toda planificación realizada para la región ha estado alineada tanto con la Estrategia Nacional de Turismo 2012-2020 como con el Plan Nacional de Desarrollo Turístico Sustentable 2015-2018. Es necesario considerar que la mayoría de estos planes, políticas y estrategias son reeditados en cada período de gobierno tomando en consideración las orientaciones estratégicas centrales y los avances en el turismo regional, por lo cual no significan cambios sustanciales.

Por otra parte, la Corporación Nacional de Fomento (CORFO) ha sido una de las instituciones que más ha invertido en el desarrollo turístico de la región. En 2007 creó un Programa Territorial Integrado para el Desarrollo del Turismo de Intereses Especiales que se extendió por cinco años. Luego, en 2009, creó un Programa de Mejoramiento de la Competitividad en Turismo. En 2016, aprobó el Programa Estratégico Nacional de Turismo Sustentable: Transforma Turismo, cuyo objetivo fue impulsar el mejoramiento de la productividad del turismo sustentable. Enmarcado en este programa nacional, son creados el Programa Estratégico Regional llamado "Arica Siempre Activa" y un Programa Territorial Integrado (PTI) llamado "En la ruta de la Sierra del Qhapaq Ñan" (2018presente). El primero de ellos, localiza su acción en el borde costero mientras que el segundo se focaliza en tres pueblos del interior, en los cuales se pueden encontrar vestigios del Qhapaq Ñan. De esta forma, Arica y Parinacota se encuentran en los programas nacionales de turismo y, si bien existe una relación con los pueblos del interior, esta es indirecta.

Respecto de las comunas rurales donde se localizan las comunidades indígenas, las iniciativas han sido específicas. El PTI de Turismo de Intereses Especiales, en 2006, creó los 
Planes de Desarrollo Turístico Comunal para las comunas rurales de Camarones, Putre y General Lagos, con énfasis en el turismo de interés especial. Más recientemente, el PTI en la Ruta de Qhapaq Ñan considera la formulación de un Plan de Negocios para el tramo Socoroma-Zapahuira-Belén. A nivel municipal, ninguna de las comunas rurales cuenta con un Plan de Desarrollo Turístico y, junto con ello, sus recursos, tanto financieros como humanos, son escasos o inexistentes (Servicio Nacional de Turismo, 2018). Actualmente, el gobierno, a través del Ministerio de Relaciones Exteriores, está formulando un Plan Maestro para las comunas rurales que considera el turismo como una alternativa económica para revertir el despoblamiento de los pueblos del interior.

Es importante recalcar que el carnaval no es considerado por la mayoría de los instrumentos de planificación. El único que hace una alusión directa es el Plan de Desarrollo Turístico Comunal de Arica elaborado en 2016. En este documento es categorizado como un atractivo que expone de manera viva la diversidad cultural de la macro región andina. No obstante, desde su primera versión, la municipalidad ha destinado sus recursos humanos, materiales y monetarios a la realización del carnaval.

\section{El componente indígena en el turismo de frontera}

El turismo se transformó en una alternativa para el desarrollo de la región, porque a diferencia de otras regiones de la zona norte, la minería tiene una participación marginal en la economía local. La autoridad regional definió el turismo como uno de los ejes de desarrollo, por lo cual este ha sido diagnosticado e incorporado en las Estrategias Regionales de Desarrollo 2008-2016 y 2017-2030. Algo similar ocurre con las necesidades de la población indígena que se encuentra en las comunas rurales de la región. En la última estrategia, cuatro lineamientos pueden ser asociados directamente al desarrollo del turismo y las comunidades indígenas:

Lineamiento Estratégico CP1: Resguardar, fortalecer, proteger y rescatar el patrimonio y culturas en todos sus ámbitos, buscando poner en valor desde una perspectiva de desarrollo sostenible, con énfasis en la cultura Chinchorro, legado Andino y paisaje cultural de la Región.

Lineamiento Estratégico ED2: Potenciar el desarrollo turístico sustentable con el patrimonio natural y cultural de la Región priorizando obras de infraestructura en el borde costero y casco antiguo de la ciudad de Arica. Lineamiento Estratégico ED8: Incentivar la conciencia turística, formación, diversificación y fortalecimiento de la oferta y fomento de la promoción nacional e internacional de la Región como destino turístico.

Lineamiento Estratégico SU2: Fortalecer e incentivar el repoblamiento y disminución de migración interna desde las comunas rurales, generando condiciones económicas, de servicios básicos, conectividad, educación, reciprocidad y trabajo comunitario (Ayni), y bienestar que permitan el desarrollo de generaciones futuras mediante la cohesión territorial (Gobierno Regional de Arica y Parinacota \& PNUD, 2017, pp. 151, $154,157)$

Es posible hallar en estas estrategias regionales de desarrollo, incluso cuando la región dependía de Tarapacá, lineamientos similares respecto de la necesidad de planificar el desarrollo turístico. En este proceso, los gobiernos, de manera sucesiva, han apoyado ciertas iniciativas, principalmente aquellas asociadas a la arqueología y el patrimonio 
cultural tangible. Esta situación se sucede porque la monumentalidad y la tangibilidad representan desafíos para el desarrollo de Arica y Parinacota como destino turístico internacional.

La región se encuentra cercana a dos destinos turísticos importantes para Latinoamérica: Machu Picchu en Perú y San Pedro de Atacama en Chile. Es posible incluir también el Complejo Arqueológico de Tiwanaku en Bolivia, con lo cual se podría afirmar que la región se encuentra cercada de destinos bien posicionados. Se trata, así, de una zona de paso para los turistas que se dirigen a algunos de estos lugares. Para competir por estos visitantes, parte del presupuesto regional se ha invertido en el desarrollo de dos atractivos turísticos que tiene características similares. Uno corresponde a la puesta en valor de los Sitios Arqueológicos de la Cultura Chinchorro, que ha sido liderado por la Universidad de Tarapacá y que se ha concentrado en la elaboración del Expediente de Nominación para el Centro del Patrimonio Mundial de la Organización de las Naciones Unidas para la Educación, la Ciencia y la Cultura (UNESCO). Además, se está financiando la construcción de un museo para conservar, proteger y exponer las momias de esta cultura, que son las más antiguas del mundo. Otra inversión que se ha mantenido a través de los distintos gobiernos es la restauración de las iglesias andinas de Arica y Parinacota para la conformación de la Ruta de las Misiones desarrollada por la Fundación Altiplano. En ambas iniciativas los valores culturales y el patrimonio inmaterial de las comunidades indígenas de la región no están considerados o juegan un rol marginal.

A diferencia de lo que ocurre en otras regiones del país, como en la Araucanía (ver p. ej. De la
Maza, 2018), no existe de parte de la autoridad una concepción de desarrollo turístico en torno a las comunidades indígenas. Para la mayoría de las iniciativas públicas, los indígenas y/o sus comunidades son oferentes de servicios complementarios, como hospedaje, alimentación y otros servicios menores. La actividad turística, entonces, se transforma en una alternativa para diversificar los ingresos, pero no involucra el contacto directo entre turistas e indígenas basado en sus prácticas culturales o medioambiente. Si bien existen iniciativas en el territorio indígena de la región, escasamente se observa una construcción teórica y práctica sobre el desarrollo del turismo indígena.

Al respecto, existen pocos diagnósticos nacionales sobre turismo indígena realizados por instituciones gubernamentales. Dentro de la formulación de la hoja de ruta del Programa Transforma Turismo se identifican una serie de brechas en el turismo indígena (INFYDE ID \& SEGITTUR Turismo e Innovación, 2016). Ellas corresponden a: 1) desprotección de los valores arqueológicos, patrimoniales y culturales; 2) despoblamiento de poblados rurales; 3) falta de productos de carácter innovador; 4) ausencia de capacitación; 5) falta de asociatividad; 6) débil articulación entre servicios públicos, privados y comunidades; 7) desconocimiento de recursos turísticos; 8) dificultades para acceder a los canales de comercialización, y 9) desconocimiento de gestión de turismo comunitario. Varias de estas brechas se pueden considerar para la región de Arica y Parinacota, en particular el despoblamiento de los pueblos del interior que debilita el tejido económico y social comunitario indígena. Por lo tanto, las oportunidades ofrecidas por los proyectos de turismo están limitadas a la población que aún no se han desplazado a la ciudad de forma permanente. 
De acuerdo al Censo de Población y Vivienda de 2017 , el $96,2 \%$ de la población que dice pertenecer a un pueblo indígena vive en la comuna de Arica. Parte de los descendientes indígenas, nacionales y extranjeros, mantiene una relación con sus pueblos de origen localizados tanto en el interior de la región como en el altiplano boliviano. Este hecho se puede observar en el tránsito de indígenas hacia los pueblos del interior para participar en las festividades patronales. También se puede constatar el viaje de migrantes bolivianos para participar, por ejemplo, en comicios electorales. Lo indígena se ha filtrado en la ciudad incorporándose a comunidades formales a través de las cuales se han apropiado de diversos espacios urbanos. Uno de esos espacios es el Carnaval Andino de Arica "Con la Fuerza del Sol" que se realiza en los meses de verano.

\section{Carnaval Andino de Arica "Con la Fuerza del Sol"}

El Carnaval Andino sustituyó otro evento de similares características conocido como Ginga Ariqueña. Dicho evento fue realizado por primera vez en 1982 y era organizado en torno a agrupaciones vecinales, las cuales realizaban presentaciones con carros alegóricos imitando al Carnaval de Río. Paulatinamente, se sumaron a esta actividad organizaciones con presentaciones de las culturas andinas, quienes en 1999 decidieron retirarse de la Ginga Ariqueña para organizar sus propios eventos teniendo como festividad de referencia el Carnaval de Oruro. Sin embargo, como principal diferencia, el Carnaval Andino de Arica no responde a ciclos agrarios o religiosos, sino que se organiza como una competencia. Además, su realización se ha posicionado como la antesala a otros carnavales en los pueblos del interior y al Carnaval de Oruro.

En el caso del Carnaval de Oruro, este puede ser considerado como una muestra del folklore canónico o espectacular de Bolivia. Es decir, "un conjunto de prácticas musicales y de danza marcadamente estilizadas que han adquirido un carácter dominante y fuertemente estatuido como producto de un complejo proceso histórico de institucionalización" (Rivero Sierra, 2008, p. 109). Es necesario reconocer que este tipo de folklore fue modificado para ser aceptado por la élite no indígena y dominante. En tal sentido, otro aspecto importante de este evento es la transferencia de componentes culturales indígenas hacia el espacio urbano que permite al sujeto indígena disolver su identidad para transformarse en un indígena urbano que busca ascender en la escala social (Galinier \& Molinié, 2013). Además, desde su denominación como Obra Maestra del Patrimonio Oral e Intangible de la Humanidad por la UNESCO en 2001, como menciona Romero (2012), ha favorecido la aparición de iniciativas turísticas, pero también el vaciamiento de sus contenidos epistémicos andinos. Existieron diferentes procesos de nacionalización, popularización y legitimización relacionados con el Carnaval de Oruro en Bolivia y que no ocurrieron en Chile. En este sentido, el Carnaval Andino de Arica se nutre no tan solo de la música y la danza del carnaval de Bolivia, sino que también importó soluciones que han facilitado el desarrollo del evento, entre las que se considera su institucionalización.

Los tres entes organizadores, la Federación de la Cultura y las Artes Indígenas Kimsa Suyu, la Asociación Indígena Confraternidad 
de Agrupaciones Folclóricas Andinas de Arica Inti Ch'amampi y la Municipalidad de Arica decidieron institucionalizar el carnaval, lo que significó regularlo a través de un decreto alcaldicio ${ }^{4}$. En dicho decreto se crea un comité en el que participan representantes de cada una de las organizaciones y donde se toman acuerdos sobre diferentes ámbitos del carnaval, por ejemplo, afiche publicitario, salida de las comparsas, etc. La organización desarrolla una serie de actividades en torno al evento que responden a la tradición cultural y la promoción turística. Existen las actividades de lanzamiento, presentación de ñustas y reinas, convite, ceremonia ancestral $\left(p^{\prime} a w a^{5}\right)$ y cena de premiación. La mayoría de estas actividades son públicas y tienen como fin dar a conocer el carnaval en los diferentes medios de comunicación para atraer la mayor cantidad de turistas. Por otra parte, las comparsas tienen sus propios sistemas de organización. La mayoría considera la existencia de una o varias personalidades jurídicas, la elección de un directorio, la definición de los pasantes, la formación de comisiones, el establecimiento de reglamentos, entre otros. El alto grado de institucionalización del carnaval permite potenciar ciertos discursos, en particular el énfasis en su aporte a la ciudad y el turismo, con lo cual se han obtenido nuevos apoyos para su realización.

El Carnaval 2019 se diferencia de sus versiones anteriores por la diversificación de fuentes de financiamiento y la promoción en la capital. La Corporación Municipal de Turismo Costa Chinchorro fue capaz de apalancar recursos desde el sector privado. Igualmente, el gobierno regional destinó fondos para apoyar otras actividades del carnaval, como, por ejemplo, un patio de comidas. Con este aporte también se financió la visita al Palacio de La Moneda, donde representantes de los organizadores, políticos de la región, bailarines y músicos fueron recibidos por la ministra secretaria general de Gobierno. En dicho acto se realizó una p'awa, ceremonia que ya es tradicional entre indígenas y autoridades, y también se presentó una muestra de las danzas del carnaval, que incluyeron las de origen boliviano. Muy probablemente, sin la resignificación de este evento como parte del desarrollo turístico regional, estas expresiones culturales hubieran sido excluidas de este espacio. No obstante, esta actividad no pasó desapercibida por la prensa y el gobierno boliviano. Este último, a través del Ministerio de Culturas y Turismo, emitió un comunicado al respecto:

\begin{abstract}
Es imperante que las manifestaciones culturales expresen el respeto mutuo entre nuestros pueblos y naciones, reconociendo el origen y procedencia de danza, coreografía, música y vestuario que, en este caso, son patrimonio cultural del Estado Plurinacional de Bolivia, reconocidas por leyes nacionales y organismos internacionales (Ministerio de Culturas y Turismo de Bolivia, 2019).
\end{abstract}

Esta solicitud del reconocimiento de origen ha sido permanente por parte de las autoridades bolivianas y no solo respecto del Carnaval Andino de Arica, sino también de otros eventos culturales, como el Carnaval de Juliaca en Perú. A pesar de esta situación, por primera vez una ministra de Culturas y Turismo de Bolivia visitó el Carnaval Andino de Arica. Wilma Alanoca Mamani, invitada por las fraternidades de residentes bolivianos, participó en la ceremonia de la p'awa junto a autoridades locales y representantes de las agrupaciones de danza del carnaval. Políticamente, este hecho representa un apoyo que hace la autoridad boliviana hacia las comunidades de migrantes bolivianos en Chile. La visita de la ministra demuestra que la 
postura no es la competencia entre los carnavales de Arica y Oruro. Existe una afirmación sobre el origen de las danzas bolivianas con lo cual se le otorga un grado de independencia con el fin de salvaguardar la identidad distintiva de estas expresiones. Bolivia estaría, entonces, ejerciendo soberanía cultural al reafirmar el derecho a mantener la relación histórica tanto con las danzas como con la población boliviana que es parte de estas expresiones. Al contrario, el desarrollo turístico que proponen las autoridades locales busca mostrar al carnaval como un solo producto, lo que origina un proceso de homogeneización cultural de las diferentes danzas, sean estas locales o internacionales.

Las expresiones transnacionales e indígenas han sido invisibilizadas por este proceso. En el marco propuesto como un espectáculo y una competencia, no solo se resaltan los trajes, las coreografías y la música, sino también, estratégicamente, las nacionalidades extranjeras, más la boliviana que la peruana. Sin embargo, estas distinciones no son percibidas por los turistas y tampoco por las personas locales. El siguiente apartado busca revelar este aspecto.

\section{El carnaval en el espacio transnacional urbano}

El carnaval no solamente se puede categorizar como un espacio transnacional porque se bailan danzas y ritmos que originalmente nacieron en Bolivia, sino que existe toda una representación desde tres países que se conjuga entre sí. En este lugar, diversos actores interactúan a partir de diferentes procesos y definen la formación de poder, significados e identidades en el carnaval. En este aspecto es necesario examinar los aportes que hacen los migrantes en el desarrollo de este evento.
La región de Arica y Parinacota tiene, en particular, un valor geopolítico para Chile dado los lazos económicos, sociales y políticos que se establecieron con Bolivia y Perú. De cierta forma existe seguridad respecto de la soberanía chilena en la ciudad y las fronteras dada la presencia de diferentes organismos estatales. No obstante, en el territorio intermedio el Estado está casi ausente. Los pequeños asentamientos y pueblos localizados en los valles, la precordillera y el altiplano parecen gozar de ciertas libertades sociales y culturales. Aunque ha existido una circulación histórica de población entre los diferentes poblados de los valles, la precordillera y las montañas, una vez afianzadas las fronteras, este movimiento es reinterpretado como un fenómeno migratorio. Es así como los migrantes bolivianos, con diferentes estatus, llegan a desempeñarse en la agricultura y la ganadería de los pueblos del interior. Frente al despoblamiento que estos sufren actualmente, ocurre un tránsito de los migrantes desde la cordillera hacia la costa, en el que se asientan en particular en el valle de Azapa. La permanencia en Chile de los migrantes produce la regularización, trámite en el que algunos obtienen la residencia permanente o la nacionalización. Este proceso es difícil de dimensionar porque la mayoría de los migrantes indocumentados no se relaciona con la autoridad, por lo tanto, no participa en encuestas o censos. En este espacio, la cultura mal llamada "extranjera" comienza a afianzarse.

Es importante considerar que los migrantes bolivianos y sus descendientes son miembros de algunas comparsas del carnaval. Desde ese espacio, se desarrollan como una comunidad transnacional puesto que han sido capaces de recrear las prácticas foráneas en el espacio nacional. De esta forma, varias comparsas 
contratan bandas bolivianas y ocupan vestimentas diseñadas por costureras de Bolivia. Asimismo, ya es una tradición que algunas comparsas celebren con un pasacalle en el centro de Arica el Día Nacional de Bolivia en la semana del 6 de agosto. Estos ejemplos demuestran la conexión cultural y material que influye en la reterritorialización de las prácticas andinas en la zona urbana.

La ciudad es un espacio impermeabilizado a las manifestaciones culturales no chilenas dado los procesos de fortalecimiento de la identidad y la cultura nacional. Es, por lo mismo, que implícitamente se transforma en un espacio codiciado para los procesos reivindicatorios de los pueblos indígenas y también migrantes. Las agrupaciones de baile no se focalizan en la periferia, porque estos espacios ya están ocupados, por ejemplo, en el terminal agropecuario que se encuentra en la salida sur de Arica. Allí, la tarqueada, la morenada y otros bailes son conocidos y se complementan con el comercio de ciertos productos bolivianos, tales como, aguayos, bolsas de hoja de coca, cervezas de marcas bolivianas, entre otros. De manera intencionada, las agrupaciones de migrantes y sus descendientes que gozan de la nacionalidad chilena apuntan sus expresiones culturales hacia el centro de la ciudad. En este lugar las fraternidades logran nuevos adeptos, quienes no necesariamente comparten la identidad indígena o migrante. Llegar a ese espacio no fue posible sin resignificar los bailes como un recurso que podría incentivar el desarrollo turístico de la región.

Este proceso que desarrollan las personas con ascendencia indígena, también es compartido con otros grupos. Entre ellos destacan las comparsas de afrodescendientes. Ellas emplearon el carnaval como un espacio de reconstrucción y rescate identitario a través del cual visibilizaron el movimiento afrodescendiente en la escala regional y nacional (Espinosa, 2015). De esta forma, el carnaval contribuyó al reconocimiento que les otorgó el Estado como pueblo tribal en 2019.

El centro de Arica adquiere un simbolismo particular como herencia del conflicto y el triunfo. En el Morro de Arica, una bandera de 18 por 27 metros flamea en un mástil de 42 metros de alto que reemplazó a una anterior como parte de la celebración del Bicentenario de Chile en 2010. A sus pies, por la avenida Comandante San Martín se realizan desfiles dominicales y aquellos conmemorativos de fechas militares, como el Combate Naval de lquique (21 de mayo), el Asalto y Toma del Morro de Arica (7 de junio), el Juramento a la Bandera (10 de julio) y la Parada Militar por las Fiestas Patrias y Glorias del Ejército (18 de septiembre). Allí también se encuentran varios monumentos, como las estatuas de los héroes de la historia de Chile Bernardo O'Higgins y Arturo Prat y los bustos de militares que participaron en la Batalla de Arica, como Pedro Lagos, Juan José de San Martín, Luis Solo de Zaldívar y Ricardo Silva Arriagada. En este espacio, enaltecido por su geografía, se enardece la chilenidad y se amplifican los rituales y los estereotipos nacionales.

Los símbolos del Morro y la bandera chilena no son excluidos del carnaval, sino que ocupan un espacio especial en la promoción. La comisión organizadora solicitó a la Municipalidad de Arica poner en el centro de los afiches promocionales de los carnavales de 2018 y 2019 una foto del Morro y la bandera chilena. Cabe mencionar, sin embargo, que varios de los monumentos anteriormente mencionados parecen desapa- 
recer en el momento del carnaval ante el escenario principal y las graderías y por la atmósfera de festejo.

No obstante, en el carnaval las banderas de Chile, Bolivia y Perú se entrecruzan en las vestimentas de los bailarines y los músicos, muchas de ellas diseñadas y confeccionadas en Bolivia. Estas banderas aparecen también junto con la wiphala $^{6}$ que representa a los pueblos indígenas en Latinoamérica y que fue declarada símbolo patrio del Estado Plurinacional de Bolivia. En general, el significado de la wiphala es desconocido para los asistentes, incluso en algunos casos es confundida con la bandera de la diversidad sexual por la cantidad de colores. Algo similar sucede con la bandera blanca que utilizan los pueblos del interior en los bailes más tradicionales. Esta bandera es llevada exclusivamente por los pasantes ${ }^{7}$, hombre o mujer, y simboliza la ancestralidad entendida como pertenencia al Collasuyo ${ }^{8}$. Equivocadamente esta es confundida con la bandera de la paz por el color blanco. La bandera blanca rivaliza con la wiphala en la representatividad de los pueblos indígenas.

En el carnaval llama la atención la comparsa Waca Waca Residentes Bolivianos, que encabeza su presentación con cuatro banderas: la chilena, la boliviana, la wiphala y la bandera de Arica, la cual lleva el escudo y el emblema de armas de la ciudad. Curiosamente, el escudo y el emblema le fue conferido por el rey de España Felipe IV en 1657 y en el centro de él se encuentra una imagen que representa el monte de Potosí de Bolivia. Cabe mencionar que, en la época colonial, Arica era el puerto por donde salían los embarques de oro y plata de Bolivia hacia España.
Al igual que su símil en Oruro, el Carnaval Andino tiene un recorrido por diversas calles en el centro de la ciudad que suman aproximadamente $2 \mathrm{~km}$. Esta es una diferencia respecto de su antecesor, la Ginga Ariqueña, que solo ocupaba una avenida. Es importante destacar que las calles de Arica fueron rebautizadas con nombres de héroes de la Guerra del Pacífico, fechas de batallas y de políticos de aquellos años. Una serie de escenarios son instalados a lo largo del recorrido para animar a cada comparsa, siendo aquel ubicado a los pies del Morro el más importante. En este lugar, las comparsas presentan sus coreografías en un tiempo determinado de acuerdo con la danza ejecutada para ser evaluadas por un jurado. Es muy típico que los adeptos a una comparsa se aglomeren en las graderías para brindarle su apoyo con lo cual se crea una atmósfera muy competitiva en ciertas categorías. Al finalizar la presentación, en una pantalla gigante se presentan dos de los tres puntajes obtenidos, los cuales son aplaudidos o abucheados por los asistentes. El recorrido oficial termina en el escenario principal, pero las comparsas siguen bailando hasta llegar lo más cerca al Morro. Allí, las bandas de bronce, muchas de ellas bolivianas y peruanas, se ubican para seguir tocando, fuera de la competencia, para los bailarines, los familiares, los organizadores y el público. Para algunas personas, el Morro de Arica es resignificado como un mallku o deidad de la montaña, de acuerdo con la cosmovisión andina. En este lugar, y al igual que en el resto del recorrido, se instalan pequeñas ferias formales e informales, donde se venden comidas, juguetes, suvenires e incluso cerveza.

En esta configuración del espacio, residentes locales y turistas llegan al espectáculo. Para 
quienes no están familiarizados con el norte de Chile, el carnaval es un tipo nuevo de evento turístico. La forma tradicional empleada por los municipios para atraer turistas es la organización de festivales que replican el Festival Internacional de la Canción de Viña del Mar o el Festival del Huaso de Olmué. En oposición, el Carnaval de Arica propone una experiencia novedosa para los turistas, pero con algunas dificultades. En particular, no existen guías turísticos que ofrezcan optimizar la experiencia en un marco de tiempo limitado. A diferencia de los residentes locales, los turistas construyen la autenticidad (Lovell \& Bull, 2018) a partir de lo que observan, sin atribuir nacionalidades al espectáculo.

Las comparsas que participan en el Carnaval tienen diferentes lugares de origen. Entre ellas destacan las formadas en el valle de Azapa, donde se localizaron los migrantes bolivianos para desempeñarse en la agricultura. En este espacio se formaron diferentes agrupaciones de bailes, entre las que destacan las tarqueadas y las morenadas. Estas asumieron nombres relacionados con localidades bolivianas, por ejemplo, Morenada San Pedro de Totora, Morenada Papel Pampa, Tarqueada Villaroel, Tarqueada Curahuara de Carangas, entre otras. Por otra parte, existen agrupaciones de residentes bolivianos, las cuales se han organizado en torno a los bailes bolivianos, por ejemplo, Morenada Central Residentes Bolivianos, Waca Waca Residentes Bolivianos y Diablada Residentes Bolivianos. Un tercer grupo corresponde a las comparsas representantes de los pueblos del interior, como, por ejemplo, el Pueblo Misaña de Belén, Pueblo Jallalla Parinacota, Pueblo Hijos de Caquena, entre otras. En este contexto, de acuerdo con Chion (2013), mientras mayor es el componente ritual, estético y significativo desde las culturas andinas representadas por actores locales, mayor es la apertura de espacios transnacionales. Cabe mencionar que algunas de las comparsas integran bailarines que provienen de filiales de otras ciudades y países. Lo anterior también sucede de manera contraria, hay bailarines que viajan a Bolivia para participar en el Carnaval de Oruro o la Fiesta de Jesús del Gran Poder que se realiza en La Paz. En estos casos, los participantes se comprometen a bailar tres años consecutivos (Tassi, 2010).

La retribución por participar en el carnaval no es monetaria, porque los premios que entrega la municipalidad no se acercan a los gastos que realizan los bailarines en la compra o el arriendo de sus trajes y la contratación de la banda que los acompaña por el recorrido, entre otros gastos. Por ejemplo, en la versión 2019, la Poderosa Morenada del Valle de Azapa contrató tres bandas bolivianas: la Banda Intercontinental Poopó, la Banda Espectacular Pagador de Oruro y la Banda Espectacular de Bolivia. En su presentación, cada banda eligió un color de la bandera boliviana (rojo, amarillo y verde) en la vestimenta de sus músicos con lo cual daba la impresión de una gran bandera en el escenario principal.

Para los bailarines, el interés en participar puede ser visto desde diferentes perspectivas: para algunos es simplemente ganar la competencia mientras que para otros es mostrar sus tradiciones y reafirmar su identidad. En este aspecto, la autenticidad, sea relacionada con la cultura chilena, la indígena (aymara-quechua) o extranjera (boliviana), se conjuga con el fin de desarrollar estrategias de posicionamiento (Chamorro, 2013). Lo interesante en la participación de la ciudadanía es que, aunque existe toda una estructura para regular su funcio- 
namiento, una persona que desee participar no necesita ningún atributo especial. Todos quienes pasan por el escenario son personas corrientes, cuyas identidades y nacionalidades parecen equiparse.

El desarrollo turístico intenta apropiarse de las expresiones culturales del carnaval para empaquetarlas como un producto turístico. Sin embargo, las interacciones que ocurren en los tres días están lejos de ser completamente controladas. Las intenciones de los participantes en las comparsas, en especial la de los bailes de origen boliviano, contribuyen a enaltecer el vínculo cultural construido por indígenas y migrantes. Las manifestaciones del carnaval calzan justo con la idea que el espacio fronterizo es socialmente construido por las interacciones.

\section{Consideraciones finales}

Durante tres días, gracias al Carnaval Andino de Arica, el centro de la ciudad se transforma en un enclave multicultural que denota la relación que la ciudad tiene con los países vecinos, en especial con Bolivia. Dicho vínculo está basado en una indigeneidad transnacional que ha reinterpretado sus expresiones culturales para mantenerlas vivas en diferentes lugares. Actualmente existe un ápice cultural de Bolivia en Chile que se entremezcla con las expresiones indígenas locales, con las que comparte un mismo origen. Aunque se podría catalogar como transnacional únicamente en este evento, es necesario reconocer el grado de influencia histórica que Bolivia ha tenido sobre Arica. Lo anterior no es casual, sino que está relacionado con los hechos que involucran el comercio y la migración, y también el grado de recono- cimiento de los derechos indígenas. En este aspecto, el proceso reivindicatorio desarrollado en Bolivia con sus pueblos indígenas ha reafirmado su identidad y cultura. El carnaval ha sido un catalizador sobre formas de expresión que no son típicas en Chile.

En el caso de la región de Arica y Parinacota, al igual que en el resto de los territorios anexados tras la Guerra del Pacífico, el Estado instaló una práctica en la ciudadanía que enaltece la chilenidad a través de la gesta militar y que se materializa en las ciudades. Este hecho transformó a la ciudad en espacio de lucha para los pueblo indígenas, y también para los migrantes. Kofman (2014) menciona que es necesario considerar el conocimiento sobre cómo los migrantes entretejen relaciones individuales y redes aprovechando la estructura sociocultural de los lugares. Los migrantes bolivianos y sus descendientes han tenido un rol relevante en el desarrollo económico de la ciudad. El éxito no se traduce necesariamente en la adopción de nuevas prácticas. Por ejemplo, no es difícil encontrar familias que realizan matrimonios o funerales al estilo boliviano en Arica. En este aspecto, el carnaval encaja con la ritualidad adoptada por estas familias. Existen, además, otros elementos no analizados que también influyen en la participación en el carnaval, por ejemplo, como el de los estudiantes indígenas que quieren solicitar una beca especial, en el caso de la educación media, que requieren de un certificado que acredite prácticas culturales/rituales.

Lo que ha logrado la estrategia de turismo desarrollada por los organizadores del carnaval es naturalizar a las comunidades y los espacios transnacionales, sin tensionar la identidad y la cultura nacional. Con estas consideraciones se ha logrado permear la autoridad central que, 
por varios motivos, desconoce estas manifestaciones culturales en el espacio nacional. El transnacionalismo en los discursos y las prácticas está permitido hasta el punto en que la identidad como sentido de pertenencia no sea interpretada como contestataria a las fronteras de los Estados-nación o que construya nuevas lealtades diferentes de las afiliaciones nacionales, incluyendo las indígenas y las étnicas. Por otra parte, el desarrollo turístico que mira hacia Santiago siempre se establecerá como una barrera para mostrar la transnacionalidad de la población y el territorio, puesto que las manifestaciones asociables con otros países deben ser parcialmente negadas. Lo contrario podría suceder si se mira el Carnaval Andino de Arica como un producto turístico de escala internacional.

\section{Agradecimientos}

Este artículo se escribió gracias al apoyo del Centro de Estudios Interculturales e Indígenas-CIIR: CONICYT/FONDAP/15110006; $y$ el Proyecto FONDECYT Regular No. 1181713.

\section{Notas}

Ley 20.663, Ministerio del Interior y Seguridad Pública, "Declara Feriado el Día 7 de junio para la Región de Arica y Parinacota", 30 de abril de 2013.

2 De manera genérica se refiere a los pueblos ubicados en las áreas alejadas de la zona urbana que corresponde a la ciudad de Arica. Con algunas excepciones, los pueblos del interior tienen relación directa con comunidades indígenas.

3 "Chile Profundo" y "América Profunda" fueron conceptos utilizados por la ministra secretaria general de Gobierno y el alcalde de Arica, respectivamente, en referencia al Carnaval Andino de Arica durante la visita promocional al Palacio de La Moneda en enero de 2019. Recuperado de http://www.msgg.gob.cl/wp/index.php/2019/01/29/ ministra-cecilia-perez-recibe-a-artistas-del-carnaval-andino-dearica-ustedes-son-el-chile-profundo-representan-unidad-y-traenla-fortaleza-de-nuestra-diversidad-cultural/

4 Decreto Alcaldicio No. 942/2019, Municipalidad de Arica Aprueba bases del Carnaval con la Fuerza del Sol Año 2019, 18 de enero de 2019.
5 La p'awa es una ceremonia realizada por un yatiri (sabio indígena) que tiene como objetivo pedir una bendición de la Pachamama para la realización de una actividad específica.

6 La wiphala es una bandera basada en la simbología tradicional de las comunidades indígenas aymaras. Está organizada en 7 filas y 7 columnas que producen 49 cuadrados. De manera diagonal, se distribuyen siete colores: blanco, verde, azul, violeta, rojo, naranja y amarillo.

7 El pasante es un cargo dentro de las fraternidades o comparsas bajo el cual se asumen todos los gastos de la participación en el carnaval. Quien asume este rol, al igual que su familia, adquiere gran prestigio ante sus pares.

8 El Collasuyo era el nombre de la región andina en la América precolombina, zona donde se ubican actualmente Bolivia, el noroeste de Chile y el noroeste de Argentina. Junto con el Antisuyo, el Contisuyo y el Chinchaisuyo formaban el Tahuantinsuyo, que fue el estado teocrático con el dominio más extenso en América del Sur. 


\section{Referencias bibliográficas}

Bhabha, H. K. (2014). The Location of Culture. Nueva York: Routledge.

Brown, G. W. (2013). Diaspora, Transnationalism, and Issues in Contemporary Politics. En Quayson, A. \& Daswani, G. (Eds.), A Companion to Diaspora and Transnationalism (pp. 68-97). Londres: Wiley Blackwell.

Cadena, M. de la (2009). Política indígena: un análisis más allá de 'la política'. Red de Antropologías del Mundo - World Anthropologies Network, (4), 139-171.

Chamorro, A. (2013). Carnaval Andino en la ciudad de Arica: performance en la frontera norte chilena. Estudios Atacameños. Arqueología y Antropología Surandina, (45), 41-54.

Chang, T. C. (2012). Making and Unmaking Places in Tourism Geographies. En Wilson, J. (Ed.), The Routledge Handbook of Tourism Geographies (pp. 133-138). Londres y Nueva York: Routledge.

Chion, M. (2013). Cusco: City of Memory. En Irazábal, C. (Ed.), Transbordering Latin Americas. Liminal Places, Cultures, and Powers (T)Here (pp. 97-113). Nueva York y Londres: Routledge.

Crang, P., Dwyer, C., \& Jackson, P. (2003). Transnationalism and the Spaces of Commodity Culture. Progress in Human Geography, 27(4), 438-456.

Cuninghame, P. G. (2008). Hybridity, Transnationalism, and Identity in the US-Mexican Borderlands. En lyall Smith, K. E. \& Leavy, P. (Eds.), Hybrid Identities: Theoretical and Empirical Examinations (pp. 13-40). Leiden y Boston: Brill.

Dahinden, J. (2012). Transnational Belonging, Non-Ethnic Forms of Identification and Diverse Mobilities: Rethinking Migrant Integration? En Messer, M., Schroeder, R. \& Wodak, R. (Eds.), Migrations: Interdisciplinary Perspectives (pp. 117-128). Londres: Springer.

Derichs, C. (2017). Knowledge Production, Area Studies and Global Cooperation. Londres y Nueva York: Routledge.

Djelic, M.-L., \& Quack, S. (2010). Transnational Communities and their Impact on the Governance of Business and Economic Activity. En Djelic, M.-L. \& Quack, S. (Eds.), Transnational Communities: Shaping Global Economic Governance (pp. 377-413). Nueva York: Cambridge University Press.

Espinosa, M. P. (2015). Afrochilenos en Arica: identidad, organización y territorio. Antropologías del Sur, 2(3), 175-190.

Faist, T. (2008). Migrants as Transnational Development Agents: An Inquiry into the Newest Round of the Migration-Development Nexus. Population, Space and Place, 14(1), 21-42.

(2010). Diaspora and Transnationalism: What Kind of Dance Partners? En Bauböck, R. \& Faist, T. (Eds.), Diaspora and Transnationalism: Concepts, Theories and Methods (pp. 9-34). Amsterdam: Amsterdam University Press.

(2013). Transnationalism. En Gold, S. J. \& Nawyn. S. J. (Eds.), Routledge International Handbook of Migration Studies (pp. 449-459). Londres y Nueva York: Routledge.

Fisker, J. K., Kwiatkowski, G., \& Hjalager, A.-M. (2019). The
Translocal Fluidity of Rural Grassroots Festivals in the Network Society. Social \& Cultural Geography, 1-23.

Galinier, J., \& Molinié, A. (2013). Los neo-indios: una religión del Tercer Milenio. Quito: Abya Yala.

Gavilán, V. \& Carrasco, A. M. (2009). Festividades andinas y religiosidad en el norte chileno. Chungara. Revista de Antropología Chilena, 41(1), 101-112.

Gobierno Regional de Arica y Parinacota \& PNUD (2017). Estrategia Regional de Desarrollo de Arica y Parinacota 2017-2030. Enfoque basado en el Desarrollo Humano. Gobierno Regional de Arica y Parinacota, Programa de Naciones Unidas para el Desarrollo, Arica.

INFYDE ID \& SEGITTUR Turismo e Innovación (2016). Servicio de Consultoria Experta para Identificación de Oportunidades y Levantamiento de Brechas y la Confección de la Hoja de Ruta del Programa Estratégico Nacional de Turismo Sustentable. Informe 6 Consolidado Final. INFYDE ID, SEGITTUR Turismo e Innovación, Santiago.

Jean, Y. S. (2007). Research Literature on Haitian Americans 1996-2006: Trends and Outlook. En Vera, H. \& Feagin, J. R. (Eds.), Handbooks of the Sociology of Racial and Ethnic Relations (pp. 411-426). Boston: Springer.

Junta de Adelanto de Arica (1974). Esquema para una Programación de Desarrollo del Interior del Departamento de Arica. Proposición Preliminar. Junta de Adelanto de Arica, Arica.

Kofman, E. (2014). Reviewing Theories of Gender and Migration: Perspectives from Europe and North America. En Battistella, G. (Ed.), Global and Asian Perspectives on International Migration (pp. 119-137). Nueva York, Londres y Cham: Springer.

Leal, J. (2017). Narrativas Territoriales de la Comunidad Aymara de Chapiquiña en los Altos de Arica. Revista Chilena de Antropología, (36), 234-254.

Lefebvre, H. (1991 [1974]). The Production of Space. Trad. D. Nicholson-Smith. Oxford: Blackwell.

Lovell, J., \& Bull, C. (2018). Authentic and Inauthentic Places in Tourism: From Heritage Sites to Theme Parks. Londres y Nueva York: Routledge.

Mamani, B. (2019). Carnaval activó comercio y turismo por casi un mes. La Estrella de Arica (20/2/2019).

Maza, F. de la (2018). Gobierno local, política y turismo indígena: enfoque etnográfico en contextos interculturales. Antropologías del Sur, 5(9), 199-213.

Ministerio de Culturas y Turismo de Bolivia (2019). Comunicado del Ministerio de Culturas y Turismo del Estado Plurinacional de Bolivia. @Wilma_DeEIAlto @ViceminTurismo[Tweet] (30/1/2019). Recuperado de https://twitter.com/CulturasBolivia/status/1090750423487664129.

Plüss, C. (2018). Transnational Lives in Global Cities. AMulti-Sited Study of Chinese Singaporean Migrants. Cham: Palgrave Macmillan.

Richards, G. (2017). From Place Branding to Placemaking: The Role of Events. International Journal of Event and Festival 
Management, 8(1), 8-23.

Rivero, F. (2008). Los bolivianos en Tucumán: migración, cultura e identidad. Tucumán: F. Rivero Sierra, Consejo de Investigaciones de la Universidad Nacional de Tucumán.

Romero, J. R. (2012). Colonialidad y dinámica festiva: legitimación de la modernidad/colonialidad en el Carnaval de Oruro. T'inkazos, 15(31), 137-156.

Ruiz, R. (2018). Zonas extremas continentales y política exterior: un escenario estratégico e impostergable para Chile. Diplomacia, (139), 44-51.

Servicio Nacional de Turismo (2018). Informe de Gestión 2017. Programa de Turismo Municipal. Servicio Nacional de Turismo, Santiago.

Smith, M. P. (2001). Transnational Urbanism: Locating Globalization. Oxford: Blackwell.
(2017). Explorations in Urban Theory. Londres y Nueva York: Routledge.

Tassi, N. (2010). Cuando el baile mueve montañas: religión y economía cholo-mestizas en La Paz, Bolivia. La Paz: Fundación Praia.

Theodossopoulos, D. (2010). Tourists and Indigenous Culture as Resources: Lessons from Embera Cultural Tourism in Panama. En Macleod, D. V. L. \& Carrier, J. G. (Eds.), Tourism, Power and Culture: Anthropological Insights (pp. 115-133). Bristol, Buffalo y Ontario: Channel View Publications.

Woo, E., Uysal, M., \& Sirgy, M. J. (2019). What Is the Nature of the Relationship Between Tourism Development and the Quality of Life of Host Communities? En Campón-Cerro, A. M. J., HernándezMogollón, M. \& Folgado-Fernández, J. A. (Eds.), Best Practices in Hospitality and Tourism Marketing and Management: A Quality of Life Perspective (pp. 43-62). Cham: Springer. 